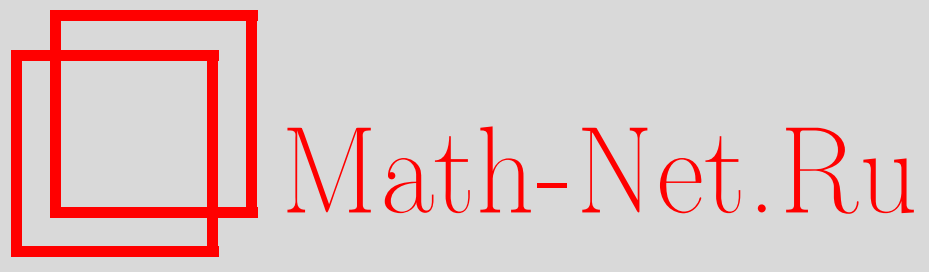

А. Р. Ярмухаметов, Гигантская и мелкие компоненты в случайных дистанционных графах специального вида, Матем. заметки, 2012, том 92, выпуск 6, 949-953

DOI: https://doi.org/10.4213/mzm10153

Использование Общероссийского математического портала Math-Net.Ru подразумевает, что вы прочитали и согласны с пользовательским соглашением http://www . mathnet.ru/rus/agreement

Параметры загрузки:

IP : 52.205 .19 .152

26 апреля 2023 г., 15:36:27

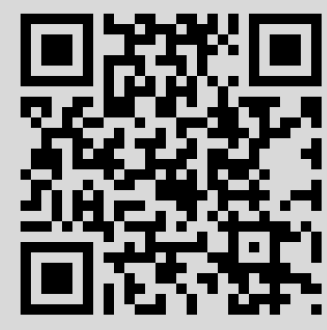




\section{Гигантская и мелкие компоненты в случайных дистанционных графах специального вида}

\section{А. Р. Ярмухаметов}

\section{1. Постановка задачи и формулировка результата}

В работе [1] была доказана теорема о существовании гигантской компоненты в случайном дистанционном графе при вероятности ребра $p=c / N$, где $c>1$. При этом также утверждалось, что "все остальные вершины содержатся в компонентах размера $o(N)$ ". В данной работе удалось показать, что "все остальные вершины содержатся в компонентах размера $O(\ln N)$ ". Этот результат является существенным продвижением, поскольку именно он является аналогом теоремы Эрдеша-Реньи для классической модели (см. [2]).

В данной работе рассматривается задача о пороговой вероятности существования гигантской компоненты для некоторой серии случайных дистанционных графов специального вида.

Положим $n=4 k, k \in \mathbb{N}, N=C_{n}^{n / 2}$ и рассмотрим полный дистанционный граф $\mathcal{G}_{N}=$ $\left(\mathcal{V}_{N}, \mathcal{E}_{N}\right)$, у которого

$$
\begin{aligned}
& \mathcal{V}_{N}=\left\{\mathbf{x}=\left(x_{1}, \ldots, x_{n}\right): x_{i} \in\{0,1\}, x_{1}+\cdots+x_{n}=2 k=\frac{n}{2}\right\}, \\
& \mathcal{E}_{N}=\left\{\{\mathbf{x}, \mathbf{y}\} \in \mathcal{V}_{N} \times \mathcal{V}_{N}:(\mathbf{x}, \mathbf{y})=x_{1} y_{1}+\cdots+x_{n} y_{n}=k=\frac{n}{4}\right\} .
\end{aligned}
$$

Таким образом, вершины полного дистанционного графа являются точками из $\{0,1\}^{n}$ и этих вершин ровно $N$. При этом ребра графа $\mathcal{G}_{N}$ суть пары его вершин, удаленных друг от друга на расстояние $\sqrt{n / 2}$. Именно этим и обусловлено название графа.

Определим вероятностное пространство - пространство случайных дистаниионных графов -

$$
\mathcal{G}^{\text {dist }}(N, p)=\left(\Omega_{N}^{\text {dist }}, \mathcal{F}_{N}^{\text {dist }}, \mathcal{P}_{N, p}^{\text {dist }}\right),
$$

где $\Omega_{N}^{\text {dist }}$ - множество всех остовных подграфов $G=\left(\mathcal{V}_{N}, E\right)$ полного дистанционного графа $\mathcal{G}_{N}, \mathcal{F}_{N}^{\text {dist }}=2^{\Omega_{N}^{\text {dist }}}$

$$
\mathcal{P}_{N, p}^{\text {dist }}(G)=p^{|E|}(1-p)^{\left|\mathcal{E}_{N}\right|-|E|}, \quad p \in(0,1) .
$$

С одной стороны, рассмотрение графов, подобных полному дистанционному графу, глубоко мотивировано задачами комбинаторной геометрии (см. [3]-[5]). С другой стороны, данная модель является обобщением классической модели случайного графа Эрдеша-Реньи (см. [2], [6]-[9]).

Будем говорить, что случайный граф обладает некоторым свойством асимптотически почти наверное (кратко а.п.н.), если вероятностная мера множества графов, обладающих этим свойством, стремится к 1 при $N \rightarrow \infty$. Отметим, что вероятность ребра $p$ есть, вообще говоря, функция от $N$.

Заметим, что граф $\mathcal{G}_{N}$ регулярный. Обозначим через $N_{1}$ степень каждой из его вершин. В соответствии с формулой Стирлинга

$$
N_{1}=\left(C_{n / 2}^{n / 4}\right)^{2}=\frac{2 \sqrt{2 \ln 2}}{\sqrt{\pi}} \cdot \frac{N}{\sqrt{\ln N}} \cdot(1+o(1)) .
$$

Сформулируем теорему о существовании гигантской компоненты в случайном дистанционном графе.

Теорема 1. Пусть $p_{*}=1 / N_{1}$. Тогда выполнены следующие утверждения: 
а) если с $>1$ и при всех $N \in \mathbb{N}$ выполнено равенство $p=c p_{*}$, то случайный граф а.п.н. содержит "гигантскую компоненту" размера

$$
N\left(1-t_{c}\right)(1+o(1)), \quad \text { где } \quad t_{c}=\frac{1}{c} \sum_{k=1}^{\infty} \frac{k^{k-1}}{k !}\left(c e^{-c}\right)^{k}
$$

при этом все остальные вершины содержатся в компонентах размера $O(\ln N)$;

б) если с $<1$ и при всех $N \in \mathbb{N}$ выполнено равенство $p=c p_{*}$, то случайный граф а.п.н. состоит из компонент, количество вершин в каждой из которых равно $O(\ln N)$.

ЗАмечАниЕ. Для величины $t_{c}$ верны следующие соотношения:

$$
t_{c}=1 \quad \text { при } c<1, \quad t_{c}<1 \quad \text { при } \quad c>1 .
$$

Доказательство этого факта можно найти, например, в [2].

Пункт б) теоремы доказан в [10]. Доказательству пункта а) теоремы 1 будет посвящен раздел 2.

\section{2. Схема доказательства пункта а) теоремы 1}

2.1. Формулировки вспомогательных утверждений и лемм. Пусть $T_{k, N}-$ число различных $k$-вершинных деревьев в полном дистанционном графе $\mathcal{G}_{N}$. Следующее утверждение является уточнением части 2 утверждения 1 из [1].

УтвеРЖДЕнИЕ 1. Пустъ $1 \leqslant k \leqslant N$. Тогда имеет место неравенство

$$
T_{k, N} \leqslant N \cdot N_{1}^{k-1} \cdot \frac{k^{k-2}}{k !} .
$$

Пусть случайная величина $U_{N, k}$ обозначает количество компонент связности размера $k$, $k \geqslant 2$, в случайном графе в пространстве $\mathcal{G}^{\text {dist }}(N, p)$. Пусть случайная величина $U_{N}(h)$ есть количество вершин в связных компонентах, размеры которых больше, чем $h \ln N$, и не превышают $N_{1}^{0,9}$. Иными словами,

$$
U_{N}(h)=\sum_{k=[h \ln N]+1}^{\left[N_{1}^{0,9}\right]} k U_{N, k}
$$

УтвеРЖДЕниЕ 2. Пусть $c \neq 1$ и $\delta(N)$ - произвольная функиия такая, ито $\delta(N)=$ $O\left(N^{-1 / 3}\right)$. Пусть при всех $N \in \mathbb{N}$ выполнено равенство $p=c(1+\delta(N)) / N_{1}$. Тогда для $q=\left(c e^{-(c-1)}\right)^{1 / 2}$ npu $h_{0}=-1 / \ln q$ a.n.н. $U_{N}\left(h_{0}\right)=0$.

Заметим, что утверждение 2 является уточнением утверждения 5 из [1].

Обозначим через $Y_{k, N}, 3 \leqslant k \leqslant\left[h_{0} \ln N\right]$, число различных $k$-вершинных компонент связности, не являющихся деревьями, в случайном графе $G$ в пространстве $\mathcal{G}^{\text {dist }}(N, p)$. Обозначим также через $Z_{N}$ число вершин, содержащихся в таких компонентах, т.е.

$$
Z_{N}=\sum_{k=3}^{\left[h_{0} \ln N\right]} k Y_{k, N}
$$

Имеет место следующее утверждение.

УтверЖДЕние 3. Пусть $c \neq 1$ и $\delta(N)$ - произвольная функиия такая, что $\delta(N)=$ $O\left(N^{-1 / 3}\right)$. Пусть при всех $N \in \mathbb{N}$ выполнено равенство $p=c(1+\delta(N)) / N_{1}$. Тогда а.п.н. выполнено соотношение $Z_{N}=o(\ln N)$. 
Утверждение 3 является уточнением утверждения 2 из [1].

Пусть случайная величина $X_{N, k}$ обозначает количество древесных компонент размера $k$, $k \geqslant 1$, в случайном графе в пространстве $\mathcal{G}^{\text {dist }}(N, p)$. Пусть случайная величина $X_{N}$ есть количество вершин в древесных компонентах, размеры которых не превышают $h_{0} \ln N$, т.е.

$$
X_{N}=\sum_{k=1}^{\left[h_{0} \ln N\right]} k X_{N, k} .
$$

Следующее утверждение является уточнением утверждения 3 из [1].

УтвеРЖДЕнИЕ 4. Пусть $c \neq 1$ и $\delta(N)$ - произвольная функиия такал, что $\delta(N)=$ $O\left(N^{-1 / 3}\right)$. Пусть при всех $N \in \mathbb{N}$ выполнено равенство $p=c(1+\delta(N)) / N_{1}$. Тогда имеет место равенство

$$
E X_{N}=N t_{c}+O\left(N^{0,8}\right) .
$$

Следующее утверждение является уточнением утверждения 4 из [1].

УтвеРЖДЕнИЕ 5. Пусть $c \neq 1$ и $\delta(N)$ - произвольная функиия такая, что $\delta(N)=$ $O\left(N^{-1 / 3}\right)$. Пусть при всех $N \in \mathbb{N}$ выполнено равенство $p=c(1+\delta(N)) / N_{1}$. Тогда имеет место равенство

$$
X_{N}=N t_{c}+O\left(N^{0,8}\right) .
$$

2.2. Завершение доказательства пункта а) теоремы 1. Проведем следующий трюк. Случайный выбор ребер при построении графов из пространства $\mathcal{G}^{\text {dist }}(N, p)$ с вероятностью $p=c / N_{1}$ (где $c>1$ ) проведем в два этапа. Положим

$$
p_{2}=\frac{1}{N_{1}^{4 / 3}}, \quad p_{1}=\frac{p-p_{2}}{1-p_{2}} .
$$

Заметим, что выполнены соотношения $p=p_{1}+\left(1-p_{1}\right) p_{2}$ и $p_{1}=c\left(1+O\left(N^{-1 / 3}\right)\right) / N_{1}$, а начиная с некоторого $N$, выполнено неравенство $p_{1}<p$. На первом этапе ребра дистанционного графа выбираем с вероятностью $p_{1}$, а затем те ребра, которые не были выбраны на первом этапе, выбираем с вероятностью $p_{2}$.

Проанализируем, что получается после первого этапа.

Из утверждений 3 и 5 мы знаем, что количество вершин графа в компонентах, размеры которых не превышают $h_{0} \ln N$ (где $h_{0}=h_{0}(c)$ ), а.п.н. равняется

$$
N t_{c}+O\left(N^{0,8}\right) \text {. }
$$

Следовательно, в соответствии с утверждением $2, N\left(1-t_{c}\right)+O\left(N^{0,8}\right)$ вершин содержатся в компонентах, размеры которых превышают $\left[N_{1}^{0,9}\right]$. Если эти вершины содержатся в одной компоненте, то, очевидно, они останутся в одной компоненте и после второго этапа. Предположим, что эти вершины содержатся в разных компонентах. Назовем такие компоненты плохими компонентами.

Докажем, что в таком случае после второго этапа между двумя любыми плохими компонентами будет существовать путь из ребер. Для этого рассмотрим две произвольные плохие компоненты (обозначим их $\varphi$ и $\chi$ ). Оценим сверху вероятность того, что между этими компонентами после второго этапа не существует пути.

Пусть $|\varphi|=M,|\chi|=K$.

Как было доказано в утверждении 2 из [10], число общих соседних вершин для двух любых различных вершин графа $\mathcal{G}_{N}$ при достаточно больших $N$ не меньше, чем

$$
N_{2}=10^{-4} \frac{N}{\ln N} .
$$

Обозначим через $\omega_{A, B}$ множество общих соседних вершин для вершин $A$ и $B$, где $A \in \varphi$, $B \in \chi$. Пусть также

$$
\Omega=\bigcup_{A \in \varphi, B \in \chi} \omega_{A, B}
$$


Пусть $\Omega=\left\{A_{1}, \ldots, A_{s}\right\}$. Пусть, наконец, $A_{1}, \ldots, A_{h} \in \varphi, A_{h+1}, \ldots, A_{g} \in \chi, A_{g+1}, \ldots$, $A_{s} \notin \varphi \cup \chi$.

Для каждой вершины $A_{i} \in \Omega$ введем $\alpha_{i}$ и $\beta_{i}$, обозначающие количество ребер, связывающих вершину $A_{i}$ с вершинами множеств $\varphi$ и $\chi$ соответственно. Очевидно, что

$$
\sum_{i=1}^{s} \alpha_{i} \beta_{i} \geqslant M K N_{2} \text {. }
$$

Тогда имеет место хотя бы одно из следующих неравенств:

$$
\sum_{i=1}^{g} \alpha_{i} \beta_{i} \geqslant \frac{M K N_{2}}{2}
$$

или

$$
\sum_{i=g+1}^{s} \alpha_{i} \beta_{i} \geqslant \frac{M K N_{2}}{2} .
$$

Если имеет место (1), то либо $\sum_{i=1}^{h} \alpha_{i} \beta_{i} \geqslant M K N_{2} / 4$, в таком случае

$$
\sum_{i=1}^{h} \beta_{i} \geqslant \frac{K N_{2}}{4}
$$

либо $\sum_{i=h+1}^{g} \alpha_{i} \beta_{i} \geqslant M K N_{2} / 4$, в таком случае

$$
\sum_{i=h+1}^{g} \alpha_{i} \geqslant \frac{M N_{2}}{4} .
$$

Таким образом, при достаточно больших $N$ количество ребер между вершинами множеств $\varphi$ и $\chi$ оценивается следующим образом:

$$
\operatorname{edge}(\varphi, \chi) \geqslant \min (M, K) \frac{N_{2}}{4} \geqslant N^{1,8} .
$$

Значит, вероятность того, что компоненты $\varphi$ и $\chi$ останутся несвязными после второго этапа, - это

$$
p_{1}(\varphi, \chi) \leqslant\left(1-p_{2}\right)^{\text {edge }(\varphi, \chi)} \leqslant \exp \left(-N^{0,4}\right) .
$$

Пусть теперь имеет место (2). Оценим вероятность того, что компоненты $\varphi$ и $\chi$ останутся несвязными после второго этапа следующим образом:

$$
p_{2}(\varphi, \chi) \leqslant \prod_{i=g+1}^{s}\left(\left(1-p_{2}\right)^{\alpha_{i}}+\left(1-p_{2}\right)^{\beta_{i}}-\left(1-p_{2}\right)^{\alpha_{i}+\beta_{i}}\right) .
$$

Так как для любого $i$ выполнено $\left(\alpha_{i}+\beta_{i}\right) \leqslant N_{1}$, а $p_{2}<1 / N_{1}$, то $\left(\alpha_{i}+\beta_{i}\right) p_{2}<1$. Значит, можно воспользоваться утверждением 6 из [1]. Следовательно,

$$
\begin{aligned}
p_{2}(\varphi, \chi) & \leqslant \prod_{i=g+1}^{s}\left(1-p_{2}^{2}\right)^{\alpha_{i} \beta_{i} / 3}=\left(1-p_{2}^{2}\right)^{\sum_{i=g+1}^{s} \alpha_{i} \beta_{i} / 3} \\
& \leqslant\left(1-p_{2}^{2}\right)^{M K N_{2} / 6} \leqslant\left(1-1 / N_{1}^{8 / 3}\right)^{N^{2,78}} \leqslant \exp \left(-N^{0,1}\right) .
\end{aligned}
$$

Общее количество плохих компонент при достаточно больших $N$ не больше, чем $N / N_{1}^{0,9} \leqslant$ $N^{0,2}$. Следовательно, вероятность того, что после второго этапа останется пара компонент, между которыми нет пути, оценивается так:

$$
p_{(2)} \leqslant N^{0,4} \exp \left(-N^{0,1}\right) \rightarrow 0 \quad \text { при } \quad N \rightarrow \infty .
$$


Таким образом, доказано, что после второго этапа по меньшей мере $N\left(1-t_{c}\right)+O\left(N^{0,8}\right)$ вершин содержатся в одной компоненте, назовем ее гигантской компонентой. Кроме того, из утверждений 2,4 и 5 имеем, что $N t_{c}+O\left(N^{0,8}\right)$ вершин содержатся в компонентах, размеры которых не превышают $h_{0} \ln N$. Обозначим размер самой крупной, после гигантской, компоненты через $D$. Из вышесказанного следует, что $D \leqslant O\left(N^{0,8}\right)$. Следовательно, в соответствии с утверждением $2, D=O(\ln N)$.

\section{СПИСОК ЦИТИРОВАННОЙ ЛИТЕРАТУРЫ}

[1] А. Р. Ярмухаметов, Матем. заметки, 92:3 (2012), 463-480. [2] B. Bollobás, Random Graphs, Acad. Press, New York, 1985. [3] А.М. Райгородский, Линейно-алгебраический метод в комбинаторике, МЦНМО, М., 2007. [4] А. М. Райгородский, УМН, 56:1 (2001), 107-146. [5] А. М. Райгородский, Модели случайных графов, МЦНМО, М., 2011. [6] Н. Алон, Дж. Спенсер, Вероятностный метод, Бином. Лаборатория знаний, М., 2007. [7] P. Erdős, A. Rényi, Publ. Math. Debrecen, 6 (1959), 290-297. [8] P. Erdős, A. Rényi, "On the evolution of random graphs", Magyar Tud. Akad. Mat. Kutató Int. Közl., 5 (1960), 17-61. [9] P. Erdős, A. Rényi, Acta Math. Acad. Sci. Hungar., 12 (1961), 261-267. [10] A. P. Ярмухаметов, "О связности случайных дистанционных графов специального вида", Чебышевский сб., 10:1(29) (2009), 95-108.

\section{А. Р. Ярмухаметов}

Поступило Московский государственный университет 29.12 .2011 им. М. В. Ломоносова

E-mail: ayarmuhametov@yandex.ru 